This is an Accepted Manuscript of an article published by Elsevier in Computers \& Geosciences, available online on 6 July 2015:

http://www.sciencedirect.com/science/article/pii/S009830041530 $\underline{0066}$

To cite this article: Lei Fan, Joel A. Smethurst, Peter M. Atkinson and William Powrie, 2015. Error in target-based georeferencing and registration in terrestrial laser scanning, Computers \& Geosciences, Volume 83, Pages 54-64, doi:10.1016/j.cageo.2015.06.021 


\title{
Error in target-based georeferencing and registration in terrestrial laser scanning
}

\author{
Lei Fan ${ }^{\mathrm{a}}$, Joel A. Smethurst ${ }^{\mathrm{a}}$, Peter M. Atkinson ${ }^{\mathrm{b}}$ and William Powrie ${ }^{\mathrm{a}}$ \\ ${ }^{a}$ Faculty of Engineering and the Environment, University of Southampton, University Road, \\ Southampton, SO17 1BJ, UK. \\ ${ }^{\mathrm{b}}$ Faculty of Science and Technology, Lancaster University, Lancaster, LA1 4YR, UK. \\ Corresponding author: Lei Fan (Email: lei.fan@ soton.ac.uk)
}

\begin{abstract}
Terrestrial laser scanning (TLS) has been used widely for various applications, such as measurement of movement caused by natural hazards and Earth surface processes. In TLS surveying, registration and georeferencing are two essential steps, and their accuracy often determines the usefulness of TLS surveys. So far, evaluation of registration and georeferencing errors has been based on statistics obtained from the data processing software provided by scanner manufacturers. This paper demonstrates that these statistics are incompetent measures of the actual registration and georeferencing errors in TLS data and, thus, should no longer be used in practice. To seek a suitable replacement, an investigation of the spatial pattern and the magnitude of the actual registration and georeferencing errors in TLS data points was undertaken. This led to the development of a quantitative means of estimating the registrationor georeferencing-induced positional error in point clouds. The solutions proposed can aid in the planning of TLS surveys where a minimum accuracy requirement is known, and are of use for subsequent analysis of the uncertainty in TLS datasets.
\end{abstract}

Keywords: Terrestrial Laser Scanning (TLS); Accuracy; Error; Georeferencing; Registration; Point Clouds.

\section{1: Introduction}

Terrestrial laser scanning (TLS) has been used increasingly for topographical surveying (e.g. Gallay et al., 2013), monitoring natural hazards (e.g. Jaboyedoff et al., 2012; Barnhart and Crosby, 2013) and investigating Earth surface processes (e.g. Schürch et al., 2011; Montreuil et al., 2013; Day et al., 2013). In these applications, TLS data usually need to be transformed into an external coordinate system for data fusion or the derivation of surface movement. This process is known as georeferencing. Another important process in TLS surveying is registration, which is the joining of multiple scans from different scan locations to form an integrated point cloud. An introduction on the registration methods used in TLS surveying can be found in Lichti and Skaloud (2010).

At present, the common practice for georeferencing/registration in TLS surveying is the target-based method. In this method, targets placed over a scan scene are surveyed by a scanner from successive scan locations for registration, or are measured by a second instrument for georeferencing. The instruments used for georeferencing mainly include differential global positioning systems (DGPS) and total stations. For TLS surveying in a natural environment, DGPS seems more popular (e.g. Schürch et al., 2011; Montreuil et al., 2013). 
Surface matching is another well-known georeferencing/registration method, which is usually based on the iterative closest point (ICP) algorithm developed by Besl and Mckay (1992), and Chen and Medioni (1992). An overview of the surface matching strategies can be found in Gruen and Akca (2005). The ICP method is more widely used for registration. Although some researchers (e.g. Prokop and Panholzer, 2009) have used this method to georeference multi-temporal TLS datasets, it is less popular for georeferencing, probably because of the concern that overlapping areas (required for surface matching) in sequential TLS data may have changed over time, especially in a natural environment.

Another georeferencing approach in TLS surveying is direct georeferencing. A number of researchers (Lichti et al., 2005; Mohamed and Wikinson, 2009; Reshetyuk, 2010) have investigated the accuracy of directly georeferenced TLS data. However, the use of this approach is still relatively rare in practice, probably due to its comparatively low accuracy.

Georeferencing is a crucial step for deformation measurement. Georeferencing error will result in relative positional error between multi-temporal TLS data, leading to a proportion of the detected surface variations being the georeferencing-induced error. Accurate registration is also important in TLS surveying, as registration error can cause misalignments between point clouds acquired from different scanner locations. Some empirical experiments (e.g. Bornaz et al., 2003; Schuhmacher and Böhm, 2005; Alba and Scaioni, 2007) and numerical studies (e.g. Bornaz et al., 2003; Scaioni, 2012) have been carried out to investigate the accuracy of georeferencing/registration in TLS surveying. However, assessment of TLS georeferencing/registration quality is still poorly understood (Scaioni, 2012), which is reflected by the statements in the following paragraph.

In data processing, target-based georeferencing/registration is usually carried out using software provided by scanner manufacturers. After georeferencing/registration, the software can report an estimated georeferencing/registration error, based on how well the target constraints are matched. For example, Leica Cyclone ${ }^{\circledR}$ reports a mean absolute error for registration and a residual error for each target constraint in its registration diagnostics report. The same or similar statistics are used by other software. It is currently common practice in the laser scanning industry to quote directly these error statistics as a quality control standard or for an uncertainty analysis. This approach has also widely been adopted by researchers (e.g. Barnhart and Crosby, 2013, Lague et al., 2013; Montreuil et al., 2013; Day, et al., 2013). They are single and spatial uniform statistics per point cloud. Although TLS users may have appreciated from their experience that these statistics are not adequate descriptors, the statistics are still used routinely, probably because there are no alternative suitable solutions in the literature.

A part of this paper is devoted to demonstrating that the statistics used routinely in current practice are incompetent measures of the actual georeferencing/registration-induced positional error in TLS point clouds. To seek a suitable replacement, the spatial pattern and the magnitude of the georeferencing/registration-induced positional error in point clouds were explored using numerical simulations in this paper. Based on the simulation results, a set of equations were proposed for estimation of the georeferencing/registration-induced error in point clouds. These equations provide a quantitative means of estimating the georeferencing/registration-induced positional error in TLS data points.

Although the target arrangement strategy for a higher georeferencing/registration accuracy is well appreciated in engineering surveying, it is not always possible to achieve an optimal target arrangement due to site constraints. The solutions proposed in this paper provide a simple tool for assessing if the target arrangement is acceptable for a given accuracy requirement associated with TLS data points. It also enables the analysis of the trade-off between the factors 
affecting the georeferencing/registration-induced positional error. Hence this paper can serve as a useful reference for TLS survey planning. It is also of use to subsequent analysis of positional uncertainty in TLS data points. Unless clearly specified, georeferencing/registration in the rest of this paper refers to target-based georeferencing/registration.

\section{Methods}

\subsection{Coordinate transformation problem}

Target-based georeferencing/registration involves two steps: (i) estimation of the transformation parameters based on control/tie points of known correspondences, and (ii) application of transformation to point clouds. In the context of TLS surveying, a rigid body transformation is usually used. If there is any reason to believe a scale difference is present, a similarity transformation can be used. In this paper, only the rigid body transformation is considered. This operation is expressed in Equation (1), in which the point clouds in Space B are transformed into Space A using the transformation parameters $\mathbf{R}$ and $T$.

$$
A_{i}=\mathbf{R} B_{i}+T
$$

where $A_{i}$ and $B_{i}$ represent the same points in Space A and Space B, respectively; $\mathbf{R}$ is the rotation matrix; $T$ is the translation vector.

The transformation parameters are estimated by minimising the squared differences shown in Equation (2) (i.e. a least-squares approach).

$$
\varepsilon^{2}=\sum_{j=1}^{n}\left\|A_{j}-\left(\mathbf{R} B_{j}+T\right)\right\|^{2}
$$

where $A_{j}$ and $B_{j}$ represent the same set of targets in Space A and Space B, respectively; $n$ is the number of target constraints; $\varepsilon^{2}$ is the squared differences to be minimised; $\mathbf{R}$ is the rotation matrix to be estimated; $T$ is the translation vector to be estimated.

Iteration is usually required for solving a non-linear least-squares problem such as that given in Equation (2). Meanwhile, closed-form solutions have been developed for estimating the transformation parameters, including the singular value decomposition method (Arun et al., 1987), the unit quaternion method (Faugeras and Hebert, 1986; Horn, 1987) and the orthonormal matrix method (Horn et al., 1988). Eggert et al. (1997) compared these closed-form algorithms and found no discernible differences in accuracy or stability for practical applications. In this paper, the Horn's unit quaternion method is used.

\subsection{Levelled point clouds}

Many latest laser scanners are equipped with an accurate dual-axis (tilt) compensator. When it is enabled during scans, the scanner automatically corrects the deviation of the scanner standing axis from the plumb line. Some researchers (Silvia and Olsen, 2012) have investigated the 
accuracy of the dual-axis compensators of several scanners. If a scanner is levelled and its dualaxis compensator is enabled, its vertical orientation is effectively plumb. This leads to a levelled point cloud.

For levelled point clouds, a 3D rigid body transformation can be simplified into a $2.5 \mathrm{D}$ case, including a $2 \mathrm{D}$ rigid body transformation (i.e. a rotation about the $\mathrm{Z}$ axis and translations along the $\mathrm{X}$ and $\mathrm{Y}$ axes) and a vertical translation. As the vertical translation can be determined by taking the average elevation differences between corresponding targets, a $2 \mathrm{D}$ transformation is effectively required for levelled point clouds. For example, levelled ScanWorlds (a ScanWorld represents all point clouds obtained from a scanner position) are the default setting for registration in Leica Cyclone ${ }^{\circledR}$.

To reduce the georeferencing/registration error, targets should be arranged in such a way that they can cover the full volume of a scan scene. However, it is usually more difficult to do so in the vertical direction due to site constraints. The use of an accurate dual-axis compensator can effectively remove such a requirement in the vertical direction. In addition, forcing scan data to be tied to a plumb vertical orientation eliminates some degrees of freedom for georeferencing/registration, and hence reduces the need for the targets for the same degree of georeferencing/registration accuracy. Therefore, it is beneficial to level a scanner and to enable its dual-axis compensator. In fact, it is common practice to enable the dual-axis compensator by professional surveyors.

\subsection{Definition of error}

The problem investigated in this paper is essentially an error propagation problem in the context of Geographic Information System (GIS). The study of errors in GIS has been extensive and diverse (e.g. Zhang and Goodchild 2002; Foody, and Atkinson, 2002). Leung et al. (2004) proposed a framework for error analysis and propagation in a measurement-based GIS (a concept proposed by Goodchild, 1999), and reviewed common techniques used for measuring GIS errors such as the covariance-based error band for the accuracy of points and planar lines and the maximal allowable limit (MAL) for positional error. An overview of error propagation in GIS can be found in Heuvelink (1998).

To avoid confusion, some of the terms used in the rest of this paper are defined in this section. As registration and georeferencing share the same mathematical operation, the following definitions are based on the term registration.

Target measurement error (TME): the positional difference between the measured coordinates of a target and the true coordinates in the scanner's coordinate system. For TLS, it is also known as the target acquisition accuracy or error (e.g. $2 \mathrm{~mm}$ at $1 \sigma$ for a Leica ScanStation C10 (Leica Geosystems, 2013)). The TME is caused and affected mainly by instrument errors (e.g. errors in a laser rangefinder and errors in the beam deflection unit and angle measurement system), environmental impacts (e.g. temperature, scattering and absorption of a laser beam propagating in air, refractive index of air), the surface characteristics of scanned objects (e.g. reflectance), and scan geometry (e.g. incidence angle).

Post-registration target difference (PTD): the positional difference between the coordinates of a target constraint after registration, in which a target constraint represents the same target in different coordinate systems (e.g. in Figure $1, \mathrm{~A}_{1}$ and $\mathrm{B}_{1}$ represent the same target, but have different coordinates because they are measured in different coordinate frames). In target-based registration, a set of target constraints (e.g. A1 and B1, A2 and B2, A3 and B3 in Figure 1) are used to estimate the transformation parameters. Due to the TME in each measured target, the 
same targets cannot be matched perfectly after registration. A PTD is the residual error for each target constraint (see Figure 1(c)), and is reported widely by commercial registration software.

Target registration error (TRE): an error statistic that takes the PTDs of all target constraints into account. The TRE is a measure of the average PTD of all target constraints. Different software systems may adopt different quantitative measures of TRE. In Leica Cyclone $^{\circledR}$, a mean absolute error (MAE) is used, which is defined as an average of the absolute PTDs (see Equation (3)). Other software may use the root mean square error (RMSE), that is, the root mean square PTD (see Equation (4)). In this paper, both MAE and RMSE are considered as measures of TRE. For clarity, the TRE-MA and the TRE-RMS are used to denote these two measures of TRE, respectively. The relationships between TRE and PTD are defined in Equations (3) and (4)

$$
\begin{aligned}
\text { TRE-MA (or MAE) } & =\frac{1}{n} \sum_{j=1}^{n}\left|\mathrm{PTD}_{i}\right| \\
\text { TRE-RMS (or RMSE) } & =\sqrt{\frac{1}{n} \sum_{j=1}^{n}\left(\mathrm{PTD}_{i}\right)^{2}}
\end{aligned}
$$

where $n$ is the number of target constraints.

Object registration error (ORE): the positional difference between the coordinates of an individual scan point after registration, and its true coordinates in the space that the scan point was transformed into. The transformation parameters estimated using a set of target constraints are unlikely to be free of error. As a result, positional errors in scan data points will be induced when the transformation parameters are applied to transform the data points. Such positional errors in data points are essentially OREs. A positional difference consists of a scalar difference (i.e. magnitude) and a direction. In this paper, only the magnitude is of interest and is represented by $|\mathrm{ORE}|$.

TRE is the statistic routinely quoted in current practice to represent |ORE|. To investigate if TRE is a useful describer of |ORE|, the relationship between these two parameters is explored in Section 3.2.

\subsection{Simulation method}

The focus of this paper is on the 2.5D case because levelled point clouds are preferred in TLS surveying (see Section 2.2), although the 3D case is also briefly investigated.

Figure 1a and $1 \mathrm{~b}$ show the same set of targets in two different 3D spaces: Space A and Space $\mathrm{B}$, in which $\mathrm{A}_{1}$ and $\mathrm{B}_{1}$ (or $\mathrm{A}_{1}^{\prime}$ after the coordinate transformation) form one of the target constraints. For ease of visualisation, only three targets are shown in plan. In the context of TLS surveying, the targets in one of the spaces will definitely be measured by a scanner. In this paper, Space $\mathrm{B}$ is chosen to be the scanner space and $\mathrm{B}_{i}(i=1,2,3)$ represents the coordinates measured by a scanner. Space A can be the space of the following instruments: a scanner (for registration), a DGPS or a total station (the latter two for georeferencing).

The simulations cannot be carried out without predefined characteristics of target measurement errors (TMEs). It is therefore necessary to characterise the TMEs used in the simulations. In this paper, the following assumptions are made: (1) the TME of an individual 
target (e.g. target $\mathrm{B}_{1}$ ) is a random variable obeying the normal distribution $\mathrm{N}(0, \sigma)$; (2) The TME of an individual target is isotropic; (3) The TMEs of the targets in the same space (e.g. targets $\mathrm{B}_{1}, \mathrm{~B}_{2}$ and $\mathrm{B}_{3}$ in Space $\mathrm{B}$ ) are independent of each other and have an equal standard deviation $\left(\sigma_{\mathrm{A}}\right.$ and $\sigma_{\mathrm{B}}$ for the targets in Spaces $\mathrm{A}$ and $\mathrm{B}$, respectively); (4) the TMEs of the targets in Space A are independent of those in Space B (in fact, they are unlikely to be correlated). Although more general cases involving dependency and different variances can readily be included in the simulations, these are not considered in this paper. A discussion on the effect of correlation between measurement errors in the same space is provided in Section 5.

To enable the simulations, quantitative information on TMEs is required. The typical target acquisition error is $2 \mathrm{~mm}$ (standard deviation) (Leica Geosystems, 2013). The measurement accuracy of an accurate DGPS is approximately $10 \mathrm{~mm}$. For ease of demonstration, most examples presented in the latter sections are based on $\sigma_{\mathrm{A}}=10 \mathrm{~mm}$ and $\sigma_{\mathrm{B}}=2 \mathrm{~mm}$. However, more general cases involving different $\sigma_{\mathrm{A}}$ and $\sigma_{\mathrm{B}}$ can be considered using the solutions presented in Sections 3.3.4 and 3.4.

Prior to the simulations, it is essential to create a scan object in Space B. This object is used to aid in the evaluation of $|\mathrm{ORE}|$ and the illustration of its spatial pattern. As its shape and size do not affect the behaviour of |ORE|, it is specified as a cube of side length of $100 \mathrm{~m}$, which reflects the useful scan ranges for many short-range terrestrial laser scanners. The scan object in Space B is then discretised into small cubic elements, with the nodes of each element being equivalent to data points in a point cloud. These elements form a cloud of points free of positional error in Space B. A counterpart error-free dataset is also created for Space A in the same fashion and serves as the reference dataset. If the measurements of the targets in both spaces are free of error, a rigid body transformation will lead to a perfect match between data points in Space B and those in Space A. However, after the TMEs being added into each target, the transformation will cause positional errors in the data points. These errors form the basis of the analyses in this paper.

So far, the essential information required for the simulations was presented. In the following sections, the simulations are described. The simulations were divided into two parts: (1) analysis of single realisation, and (2) analysis of repeated realisations. All the simulations were carried out in MATLAB ${ }^{\circledR}$.

\subsubsection{Single realisation}

This part is devoted to addressing whether the error statistics (i.e. TRE-MA or TRE-RMS) used in current practice could reasonably represent the actual georeferencing/registration error (i.e. |ORE|) in TLS survey data, based on analyses of the outcomes of individual single realisations stated below.

A single realisation includes the following steps: (1) the number of targets and their locations are specified in Spaces A and B; (2) each target location (in Spaces A and B) is assigned a TME drawn from the pre-defined normal distributions; (3) a rigid body transformation is carried out; (4) the dataset transformed from Space A to Space B is compared to the reference dataset in Space B. This single realisation is equivalent to a registration routinely implemented in practice but has the advantage of dealing with the measurement error and the registration error in a controllable way.

For each single realisation, the TRE (TRE-MA and TRE-RMS) is calculated and is then compared to the average |ORE| of all data points over the whole spatial domain specified. This aims to assess the correlation between the TRE and the $|\mathrm{ORE}|$ at an average level. The 
calculation of the average $|\mathrm{ORE}|$ is based on the same averaging techniques used for the TRE. ORE-MA is defined as the average value of the |ORE $\mid$ of all the data points; ORE-RMS is defined as the root mean square $|\mathrm{ORE}|$ of all the data points. In addition, the correlation between the TRE and the |ORE $\mid$ of an individual data point is assessed.

\subsubsection{Repeated realisations}

The second part aims to investigate the expected value and a statistical upper limit of |ORE|, including its spatial variation and the factors affecting it. In the rest of this paper, $\mathrm{E}(|\mathrm{ORE}|)$ is used to represent the expected value of |ORE|. The single realisation presented in Section 2.4.1 is repeated 100,000 times. At the location of each data point, 100,000 realisations of positional error are formed. This information is used to calculate the $\mathrm{E}(|\mathrm{ORE}|)$ and the upper limit of $|\mathrm{ORE}|$ at each data point.

\section{Results}

\subsection{Test of codes}

Prior to the simulations, the MATLAB ${ }^{\circledR}$ code used for the coordinate transformations in the simulations was tested against Leica Cyclone ${ }^{\circledR}$. Targets obtained from several real TLS surveys were registered using Leica Cyclone $^{\circledR}$ and the MATLAB ${ }^{\circledR}$ code separately. The registration results obtained using the code were compared to those reported in Leica Cyclone ${ }^{\circledR}$, including the transformation parameters and the registration error statistics. For the targets tested, the registration results obtained through these two means were found to be the same, which justified the use of the code for the transformations in the simulations.

\subsection{Current practice}

Figure 2a illustrates three targets used for a 2D rigid body transformation. 10,000 single realisations were carried out and the result (i.e. TRE-MA/-RMS and ORE-MA/-RMS) for each of these single realisations is shown as a dot in Figures $2 b$ and $2 c$. Based on a visual examination, the distributions of the results show a lack of correlations between the ORE-MA and the TRE-MA, and between the ORE-RMS and the TRE-RMS.

The targets shown in Figure 2a were also used to investigate the relations between the TRE (-MA and -RMS) and the $|\mathrm{ORE}|$ of individual scan points. As any scan point could be used for this purpose, the one coinciding with one of the targets was used for demonstration. The simulation results in Figure 3 show a lack of correlation between the $|\mathrm{ORE}|$ of individual scan points and the TRE (TRE-MA and TRE-RMS).

The investigations presented in the preceding two paragraphs were also carried out for the case of a 3D rigid body transformation. The results are shown in Figure 4 and Figure 5, and the behaviour was similar to that observed for a 2D transformation. TLS users might have used the maximum value of PTDs (i.e. residual errors of target constraints after registration) as a measure of registration error, and might argue that it would be conservative. The relations between the $|\mathrm{ORE}|$ (on average and at individual scan points) and the maximum value of PTDs were also investigated, and a lack of correlations was found.

For ease of demonstration, two examples were used in this section. However, other cases involving more targets and different target arrangements were also investigated and similar 
behaviour was observed. The results presented in this section show that the statistics routinely used in current practice are incompetent measures of the georeferencing/registration-induced positional error in scan points. Therefore, it is recommended that these statistics are no longer used for this purpose.

\subsection{E(|ORE|) for 2D transformation}

$\mathrm{E}(|\mathrm{ORE}|)$ and its spatial pattern for a 2D rigid body transformation are presented in this section. The factors affecting $\mathrm{E}(|\mathrm{ORE}|)$ were investigated separately, leading to a generic solution to $\mathrm{E}(|\mathrm{ORE}|)$.

\subsubsection{Spatial pattern of $\mathrm{E}(|\mathrm{ORE}|)$}

The demonstration is based on some examples. In Figure 6a, the three dots and the triangle represent the targets and their centre of mass $(\mathrm{CoM})$, respectively. The simulations introduced in Section 2.4.2 were carried out to derive the $\mathrm{E}(|\mathrm{ORE}|)$, which showed a circular contour and increased with increasing horizontal distance from the CoM where the $\mathrm{E}(|\mathrm{ORE}|)$ was minimal (Figure 6a). The horizontal distance is the projected distance of a 3D point onto the $X Y$ plane. As the pattern was isotropic, it is more convenient to show it using the plot in Figure $6 \mathrm{~b}$. In the rest of this paper, most of the results are presented in this fashion. The magnitude of $\mathrm{E}(|\mathrm{ORE}|)$ is not elaborated here and a detailed demonstration on it unfolds in later sections.

The spatial pattern for different target arrangements were also investigated, two of which are shown in Figure 7. It was found that the minimum value of $E(|\mathrm{ORE}|)$ always occurred at the $\mathrm{CoM}$ of the targets. However, the magnitude of this minimum value varied with the number of targets, as demonstrated in Section 3.3.3.

A rigid body transformation consists of two parts: rotation and translation. Hence the error in this transformation has two parts (i.e. rotational error and translational error). It would be straightforward to understand that the rotational error is dependent on the distance to the CoM while the translational error is spatially uniform. As the rotational error at the CoM was zero, the minimum value of $\mathrm{E}(|\mathrm{ORE}|)$ should be the translational error.

\subsubsection{Effect of target distribution}

Prior to presenting the results, the following two parameters are introduced, with the aid of the illustration in Figure 8. The first parameter $\left(S_{\mathrm{h} i}\right)$ is the horizontal distance (i.e. the distance projected onto $X Y$ plane) from targets to their CoM. The second parameter $\left(D_{\mathrm{h}}\right)$ is the horizontal distance of a data point from the CoM of the targets.

Figure 9a-c shows three targets being arranged at different distances to their CoM and having a different layout in each case. A detailed investigation on the $\mathrm{E}(|\mathrm{ORE}|)$ for these three cases was carried out. It was found that the root mean square horizontal distances (represented by $S_{\mathrm{h}}$ ) of the targets to their CoM can be used to normalise $D_{\mathrm{h}}$ so that the $\mathrm{E}(\mid \mathrm{ORE})$ for these cases followed the same pattern. $S_{\mathrm{h}}$ can be calculated using Equation (5). Figure $9 \mathrm{~d}$ shows the $\mathrm{E}(|\mathrm{ORE}|)$ against the normalised horizontal distance $\left(D_{\mathrm{h}} / S_{\mathrm{h}}\right)$ for the three cases. After the normalisation, the curve for Case $\mathrm{c}$ (the case shown in Figure 9c) overlaid a proportion of that for Case a, so did Case b. The curve for Case $\mathrm{c}$ was much shorter than that for Case a, simply because the $S_{\mathrm{h}}$ for Case c was larger. 


$$
S_{\mathrm{h}}=\sqrt{\frac{1}{n} \sum_{i=1}^{n} S_{\mathrm{h} i}^{2}}
$$

where $n$ is the number of target constraints.

It was found that the normalisation always led to the same behaviour as that shown in Figure 9 no matter how these targets were arranged in different shapes and distances. Interestingly, the minimum value of $\mathrm{E}(|\mathrm{ORE}|)$ was independent of the target arrangements when the TMEs were the same. Although three targets were used for the demonstration in this section, more general cases involving more targets were also investigated. The same phenomenon was observed.

\subsubsection{Effect of target numbers}

The analysis reported in Section 3.3.2 was applied to different number of targets. As it would be rare to use more than nine targets for georeferencing/registration in TLS surveying, the maximum number of targets considered in the simulations was nine. The results are shown in Figure 10. The $\mathrm{E}(|\mathrm{ORE}|)$ decreased with increasing number of target constraints, although the reduction was less significant when the number of targets became larger.

It would be interesting to investigate if it is possible to normalise the $\mathrm{E}(|\mathrm{ORE}|)$ against the number of target constraints. This requires an understanding on how the number of target constraints affected the $\mathrm{E}(|\mathrm{ORE}|)$. To investigate this, the $\mathrm{E}(|\mathrm{ORE}|)$ at $D_{\mathrm{h}} / S_{\mathrm{h}}=0$ and $D_{\mathrm{h}} / S_{\mathrm{h}}=5$ for the number of target constraints considered was extracted from Figure 10, and was shown in Figure 11a and $\mathrm{b}$, respectively. Based on a least-squares method, curves were used to fit the $\mathrm{E}(|\mathrm{ORE}|)$ for both cases and the associated equations were shown in Figure 11. The $\mathrm{E}(|\mathrm{ORE}|)$ was found to be proportional to $n^{-0.5}$ (i.e. $1 / \sqrt{n}$ ), where $n$ is the number of target constraints. Therefore, it is possible to relate the $\mathrm{E}(|\mathrm{ORE}|)$ for the cases where $n>3$ to that for $n=3$, which is presented in Section 3.3.4.

\subsubsection{Normalised chart for $\mathrm{E}(|\mathrm{ORE}|)$}

So far, all the results are based on the measurement errors $\sigma_{\mathrm{A}}=10 \mathrm{~mm}$ and $\sigma_{\mathrm{B}}=2 \mathrm{~mm}$ specified in Section 2.4. To make the results more generic, an attempt was made to normalise the $\mathrm{E}(|\mathrm{ORE}|)$ by the magnitudes of the target measurement errors. It was found that the $\mathrm{E}(|\mathrm{ORE}|)$ was in proportion to the root mean square measurement errors (i.e. $\sqrt{\sigma_{\mathrm{A}}^{2}+\sigma_{\mathrm{B}}^{2}}$ ). In combination with the previous findings, the normalised expected value of $|\mathrm{ORE}|$ (i.e. E $|\mathrm{ORE}| / \sqrt{\sigma_{\mathrm{A}}^{2}+\sigma_{\mathrm{B}}^{2}}$ ) against the normalised horizontal distance (i.e. $D_{\mathrm{h}} / S_{\mathrm{h}}$ ) is shown in Figure 12.

A curve was used to fit the result for the case where $n=3$, which was based on a leastsquares method. The associated equation is given in Figure 12. The differences (i.e. residuals; not shown separately here) between the fitted curve (represented by the equation in Figure 12) and the simulation result was roughly 3 orders of magnitude smaller than the value of $E(|\mathrm{ORE}|)$. Therefore, the equation was an accurate descriptor of the $\mathrm{E}(|\mathrm{ORE}|)$, which was valid for $n=3$. Section 3.3.3 found that $\mathrm{E}(|\mathrm{ORE}|)$ was proportional to $1 / \sqrt{n}$. Therefore, a generic equation can be derived readily (Equation (6)), based on the equation given for $n=3$ in Figure 12 . 


$$
\frac{\mathrm{E}(|\mathrm{ORE}|)}{\sqrt{\sigma_{\mathrm{A}}^{2}+\sigma_{\mathrm{B}}^{2}}}=0.5 \frac{\sqrt{3}}{\sqrt{n}} \sqrt{\left(\frac{D_{\mathrm{h}}}{S_{\mathrm{h}}}\right)^{1.92}+2.1}
$$

\subsection{An upper limit of |ORE| for 2D transformation}

Using the simulations introduced in Section 3.3, it is possible to produce a normalised chart for a statistical upper limit of |ORE|, which can act as a conservative estimate of |ORE|. In the simulations, each scan data point has 100,000 values of $|\mathrm{ORE}|$ obtained from 100,000 realisations of a rigid body transformation. The upper limit of $|\mathrm{ORE}|$ was defined as the value above which only $1 \%$ of the 100,000 values (realisations) lie. As the possibility of having a |ORE $\mid$ greater than this value is very small, it is effectively an upper bound of |ORE (in a statistical sense) and is represented by $\mathrm{U}(|\mathrm{ORE}|)_{0.01}$ (the subscript 0.01 represents a $1 \%$ chance to have a value greater than the statistical upper limit defined). The charts for $\mathrm{U}(|\mathrm{ORE}|)_{0.01}$ are shown in Figure 13. The residuals (not shown separately here) of the fitted curves in Figure 13 were roughly 3 orders of magnitude smaller than the value of $U(|O R E|)_{0.01}$. Similarly to Section 3.3.4, a generic equation to describe the $\mathrm{U}(|\mathrm{ORE}|)_{0.01}$ is derived (Equation (7)).

$$
\frac{\mathrm{U}(|\mathrm{ORE}|)_{0.01}}{\sqrt{\sigma_{\mathrm{A}}^{2}+\sigma_{\mathrm{B}}^{2}}}=1.52 \frac{\sqrt{3}}{\sqrt{n}}\left[\left(\frac{D_{\mathrm{h}}}{S_{\mathrm{h}}}\right)^{2.25}+1.38\right]^{0.44}
$$

\subsection{ORE in the vertical translation}

It is demonstrated in Section 2.2 that, for levelled point clouds, a 2.5D transformation can be used, including a 2D rigid body transformation on the $X Y$ plane and a vertical $(Z)$ translation. The ORE caused by the former has been studied in detail in the preceding sections. In this section, the ORE generated during the vertical translation is discussed. The vertical translation can be determined by taking the average elevation differences between corresponding tie/control targets, and can be expressed using Equation (8). This means that the ORE in the vertical direction can be assessed separately.

$$
T_{Z}=\frac{1}{n} \sum_{i=1}^{n}\left(Z_{\mathrm{B} i}-Z_{\mathrm{A} i}\right)
$$

where $T_{Z}$ is the vertical translation; $Z_{\mathrm{A} i}$ and $Z_{\mathrm{B} i}$ are the elevations of targets in Space $\mathrm{A}$ and $\mathrm{B}$, respectively; $n$ is the number of target constraints.

The uncertainty in $T_{Z}$ can be solved readily using the law of error propagation. Assume a random variable $U$ is a function of $V$ (i.e. $U=f\left(V_{1}, V_{2}, \cdots, V_{m}\right)$ ). The solution to this error propagation problem is given in Equation (9).

$$
\sigma_{U}^{2}=\sum_{i}\left(\frac{\partial f}{\partial V_{i}}\right)^{2} \sigma_{i}^{2}+\sum_{i} \sum_{j}\left(\frac{\partial f}{\partial V_{i}}\right)\left(\frac{\partial f}{\partial V_{j}}\right) \sigma_{i j} \quad(i \neq j)
$$


Based on the assumptions (i.e. independence) made in Section 2.2, the second part on the right hand side of Equation (9) is removed. Also, as $T_{Z}$ is a linear combination of some random variables, $\left(\partial f / \partial V_{i}\right)^{2}$ becomes $(1 / n)^{2}$. Therefore, the error propagation solution (in terms of error variance) to the problem in Equation (8) is obtained and shown in Equation (10).

$$
\sigma_{T_{Z}}^{2}=\frac{1}{n} \sum_{i=1}^{n}\left(\sigma_{Z_{\mathrm{A} i}}^{2}+\sigma_{Z_{\mathrm{B} i}}^{2}\right)
$$

where $\sigma_{T_{Z}}^{2}$ is the error variance of $T_{Z} ; \sigma_{Z_{\mathrm{A} i}}^{2}$ and $\sigma_{Z_{\mathrm{B} i}}^{2}$ are the variances of the target measurement error in vertical direction in Spaces $\mathrm{A}$ and $\mathrm{B}$, respectively; $n$ is the number of target constraints.

Therefore, the analysis of ORE in the vertical direction can be based on the error variance given in Equation (10). If the expected value of $|\mathrm{ORE}|$ in the vertical direction is of interest, it can be calculated using the solution to the expected value of a folded normal distribution (Elandt, 1961; Leone et al., 1961). When the TME is unbiased (see the assumption made in Section 2.2), $\mathrm{E}(|\mathrm{ORE}|)$ in the $Z$ direction is equal to $\sqrt{2 / \pi} \sigma_{T_{Z}}$.

\section{Discussion}

In the paper, the $\mathrm{E}(|\mathrm{ORE}|)$ represents the probability-weighted average of all possible values of $|\mathrm{ORE}|$ at a location of interest in space. The $\mathrm{U}(|\mathrm{ORE}|)_{0.01}$ is an upper limit value of $|\mathrm{ORE}|$ at a location of interest, where the subscript indicates there is only a $1 \%$ chance to have a value (in all possible values of $|\mathrm{ORE}|)$ greater than the $\mathrm{U}(|\mathrm{ORE}|)_{0.01}$. In a $2.5 \mathrm{D}$ rigid body transformation (see Section 2.2), the coordinate transformation on the $X Y$ plane and along the $\mathrm{Z}$ direction are carried out separately. In this case, horizontal (Sections 3.3 and 3.4) and vertical (Section 3.5) georeferencing/registration-induced positional errors in TLS data can be reported separately. A combination of these two error components would require an understanding of their correlation. If the horizontal error is assumed to be independent of the vertical error, the combined error may be taken as the square root of the sum of the squares of the horizontal and the vertical errors.

The inadequacy of TRE to represent |ORE| is due to the use of PTD of target constraints after registration or georeferencing. PTD does not honour the spatial variation of the registration and georeferencing errors in point clouds, nor is it capable of representing the real effects of TME on registered or georeferenced point clouds.

There is a common inappropriate manipulation during registration. When a target constraint is found with a larger than average error (i.e the PTD of a target constraint is larger than the TRE), data analysts tend to disable or remove this target constraint to reduce the value of the TRE. This action is indeed very likely to reduce the value of the TRE. However, according to Section 3.2, the |ORE| was not correlated with the TRE, nor with the PTD. Meanwhile, removing a target will increase the $\mathrm{E}(\mathrm{ORE})$ (see Section 3.3.3). Therefore, this action can lead to a risk of an increase in the $|\mathrm{ORE}|$ rather than a decrease. Therefore, such manipulation is not recommended unless there is any reason to believe that the measurement of one or both of the targets in a constraint is much less accurate (e.g. a gross error) than that of the other measured targets. 
The minimum values of the statistical measures of $|\mathrm{ORE}|$ (i.e. $\mathrm{E}(|\mathrm{ORE}|)$ and $\mathrm{U}(|\mathrm{ORE}|)_{0.01}$ ) occur at the CoM of targets. However, this does not mean that the minimum value of $|\mathrm{ORE}|$ in a single realisation (i.e. a georeferencing/registration) also occurs at the CoM of targets. According to the simulation results (not reported in detail in this paper), the minimum value of |ORE| was generally not at the CoM but moving spatially from one simulation to the other. This is because the translational error in a single realisation could force the location of the minimum value of $|\mathrm{ORE}|$ to move away from the CoM.

On one hand, increasing $S_{\mathrm{h}}$ can reduce $\mathrm{E}(|\mathrm{ORE}|)$; on the other hand, the target acquisition errors (i.e. TMEs) may deteriorate with the scan distance, leading to an increase in $\mathrm{E}(|\mathrm{ORE}|)$. Equations (6) and (7) can be used to analyse the trade-off between the distribution of targets and the target acquisition errors.

The study outcomes highlight a practical concern over the georeferencing of TLS data obtained using a long range scanner capable of measuring objects beyond $1 \mathrm{~km}$. As the scan objects are far from the scanner location or may be inaccessible, TLS users may place targets around the scanner rather than on the scan objects. In this case, the $D_{\mathrm{h}} / S_{\mathrm{h}}$ ratio is large and a relatively large georeferencing error in TLS data is likely to occur. To reduce the georeferencing error, more control points are required.

The results reported in this paper are based on the assumption that the TMEs in the same space are independent of each other. DGPS measurements are usually temporally correlated within a window of time, which leads to a correlation both in magnitude and direction between the positional errors of DGPS measurements. This correlation can be avoided by taking individual measurements at a time interval longer than the window of time in correlation, which varies from one device to another. However, the correlation may be beneficial in the case where targets could not be arranged widely to encircle the whole scan area due to site constraints, as explained in the following. One can readily infer that an increase in the correlation is likely to lead to an increase in the translational error and a decrease in the rotational error, because the correlation in positional errors could force the measurement errors to occur in a similar direction. As the rotational error increases rapidly with increasing normalised distances from the $\mathrm{CoM}$ of targets, the decrease in the rotational error can outweigh the increase in the translation error beyond a threshold $D_{\mathrm{h}} / S_{\mathrm{h}}$. Hence the correlation may reduce the georeferencing error at locations beyond the threshold $D_{\mathrm{h}} / S_{\mathrm{h}}$.

This paper does not provide a detailed investigation of 3D cases, as levelled point clouds (i.e. 2.5D cases) are preferred (see discussion in Section 2.2) for TLS surveys. However, the 3D cases were explored briefly using the same simulation approach. Based on the simulation results, it seems that the $\mathrm{E}(|\mathrm{ORE}|)$ forms a group of ellipsoidal equivalence surfaces (i.e. an ellipsoidal pattern), with its minimum value occurring at the CoM of the targets. Similarly to those shown in Figure 12, the $\mathrm{E}(|\mathrm{ORE}|)$ experiences a nonlinear increase with the distance from the CoM of the targets. However, such an increase is anisotropic.

TLS has been used widely for topography surveys and measurement of terrain surface deformations of various phenomena including landslides, mining-induced subsidence, coastal recession and river bed erosion. In these applications, an understanding of the TLS data quality is essential. The methods proposed in this paper offer TLS surveyors a simple tool for evaluating the georeferencing/registration-induced uncertainty in TLS data points. The methods also provide a quantitative approach to evaluate the combined effects of survey topology and target number on the registration or georeferencing accuracy, and to assess the trade-off between these two factors. Such a quantitative evaluation is of use in the planning TLS surveys. In addition to the georeferencing/registration-induced uncertainty, the quality of TLS data can 
be affected by the presence of ground vegetation. The vegetation effects must be taken into account when using TLS in an attempt to survey vegetated ground surfaces (Fan et al, 2014).

\section{Conclusion}

This paper shows that there is a lack of correlation between the georeferencing/registrationinduced positional error (i.e. ORE) in simulated scan data and the statistics used routinely in current practice, consisting of the target georeferencing/registration error (i.e. TRE) and the residuals of target constraints after georeferencing/registration (i.e. PTD). The findings confirm that these statistics are incompetent measures of the georeferencing/registration error in point clouds acquired from TLS surveys. Therefore, it is strongly recommended that such statistics should no longer be used as quality measures of the georeferencing/registration-induced error in TLS data points.

This paper proposes solutions for the expected value (Equation (6)) and the upper limit (Equation (7)) of the georeferencing/registration-induced positional error (i.e. $|\mathrm{ORE}|$ ) in point clouds for a $2 \mathrm{D}$ rigid body transformation in the $X Y$ plane. The method of assessing the vertical transformation error was also presented (see Section 3.5). A combination of these two provides a useful tool for estimation of the positional error in TLS data (point clouds) caused by uncertainty in georeferencing/registration.

\section{Acknowledgements}

The authors acknowledge funding for the research from the UK Engineering and Physical Sciences Research Council (EPSRC) under grant number EP/G056102/1.

\section{References}

Alba, M. and Scaioni, M., 2007. Comparison of techniques for terrestrial laser scanning data georeferencing applied to 3-D modelling of cultural heritage. In: Proceedings of ISPRS Workshop 3D-ARCH 2007, "3D Virtual Reconstruction and Visualization of Complex Architechtures", ISPRS Archives - Volume XXXVI-5/W47, Zurich, Switzerland, 12-13 July, 2007.

Arun, K.S., Huang, T.S., and Blostein, S.D., 1987. Least-squares fitting of two 3D point sets. IEEE Transactions on Pattern Analysis and Machine Intelligence 9(5), 698-700.

Barnhart, T. and Crosby, B., 2013. Comparing two methods of surface change detection on an evolving thermokarst using high-temporal-frequency terrestrial laser scanning, Selawik river, Alaska. Remote Sensing, 5(6), 2813-2837.

Besl, P.J. and McKay, H.D., 1992. A method for registration of 3-D shapes. IEEE Transactions on Pattern Analysis and Machine Intelligence, 14(2), 239-256.

Bornaz, L., Lingua, A. and Rinaudo, F., 2003. Multiple scan registration in LIDAR close-range applications. In: Proceedings of ISPRS Workshop WG V/4, "Vision Techniques for Digital Architectural and Archaeological Archives", ISPRS Archives - Volume XXXIV-5/W12, Ancona, Italy, 1-3 July, 2003.

Chen, Y., and Medioni, G., 1992. Object modelling by registration of multiple range images. Image and Vision Computing, 10(3), 145-155.

Day, S.S., Gran, K.B., Belmont, P. and Wawrzyniec, T., 2013. Measuring bluff erosion part 1: terrestrial laser scanning methods for change detection. Earth Surface Processes and 
Landforms, 38(10), 1055-1067.

Eggert, D.W., Lorusso, A. and Fisher, R.B., 1997. Estimating 3-D rigid body transformations: a comparison of four major algorithms. Machine Vision and Applications, 9(5-6), 272-290.

Elandt, R.C., 1961. The folded normal distribution: two methods of estimating parameters from moments. Technometrics, 3(4), 551-562.

Fan, L., Powrie, W., Smethurst, J.A., Atkinson, P.M. and Einstein, H., 2014. The effect of short ground vegetation on terrestrial laser scans at a local scalE. ISPRS Journal of Photogrammetry and Remote Sensing, 95, 42-52.

Faugeras, O.D. and Hebert, M., 1986. The representation, recognition, and locating of 3-D objects. The International Journal of Robotics Research, 5(3), 27-52.

Foody, G.M. and Atkinson, P.M. 2002. Uncertainty in Remote Sensing and GIS: Fundamentals, published by Wiley, England.

Gallay, M., Lloyd, C.D., McKinley, J. and Barry, L., 2013. Assessing modern ground survey methods and airborne laser scanning for digital terrain modelling: a case study from the Lake District, England. Computers \& Geosciences, 51, 216-227.

Goodchild M.F., 1999. Measurement-based GIS. In: Shi W et al. (eds), Proceedings, International Symposium on Spatial Data Quality. Hong Kong: Hong Kong Polytechnic University, 1-9

Gruen, A. and Akca, D., 2005. Least squares 3D surface and curve matching. ISPRS Journal of Photogrammetry and Remote Sensing, 59(3), 151-174.

Heuvelink, G.B.M., 1998. Error Propagation in Environmental Modelling with GIS. Taylor\&Francis, London.

Horn, B.K.P., 1987. Closed-form solution of absolute orientation using unit quaternions. Journal of the Optical Society of America A-4(4), 629-642.

Horn, B.K.P., Hilden, H.M., and Negahdaripour, S., 1988. Closed-form solution of absolute orientation using orthonormal matrices. Journal of the Optical Society of America A-5(7), 1128-1135.

Jaboyedoff, M., Oppikofer, T., Abellán, A., Derron, M.H., Loye, A., Metzger, R. and Pedrazzini, A., 2012. Use of LIDAR in landslide investigations: a review. Natural Hazards, 61(1), 5-28.

Lague, D., Brodu, N. and Leroux, J., 2013. Accurate 3D comparison of complex topography with terrestrial laser scanner: application to the Rangitikei canyon (N-Z). ISPRS Journal of Photogrammetry and Remote Sensing, 82, 10-26.

Leica Geosystems, 2013. Leica ScanStation C10 Product Specifications [online], Leica

Geosystems AG, Switzerland. http://hds.leica-

geosystems.com/downloads123/hds/hds/ScanStation\%20C10/ brochuresdatasheet/Leica_ScanStation_C10_DS_en.pdf [Accessed 16 Oct 2013].

Leone, F.C., Nottingham, R.B. and Nelson, L.S., 1961. The folded normal distribution. Technometrics, 3(4), 543-550.

Leung, Y., Ma, J.H., and Goodchild, M.F., 2004. A general framework for error analysis in measurement-based GIS Part 1: The basic measurement-error model and related concepts. Journal of Geographical Systems, 6, 325 - 354.

Lichti, D., Gordon, S., and Tipdecho, T., 2005. Error models and propagation in directly georeferenced terrestrial laser scanner networks. Journal of Surveying Engineering, 131(4), $135-142$.

Lichti, D. and Skaloud, J., 2010. Registration and calibration (Chapter 3), in Airborne and Terrestrial Laser Scanning, edited by Vosselman, G. and Maas, H.G., Whittles Publishing, 
ISBN: $190444587 X$.

Mohamed, A. and Wikinson, B., 2009. Direct georeferencing of stationary LiDAR. Remote Sensing, 1(4), 1321-1337.

Montreuil, A.L., Bullard, J.E., Chandler, J.H. and Millett, J., 2013. Decadal and seasonal development of embryo dunes on an accreting macrotidal beach: North Lincolnshire, UK. Earth Surface Processes and Landforms, 38(15), 1851-1868.

Prokop, A. and Panholzer, H., 2009. Assessing the capability of terrestrial laser scanning for monitoring slow moving landslides. Natural Hazards and Earth System Sciences, 9, 19211928.

Reshetyuk, Y., 2010. Direct georeferencing with GPS in terrestrial laser scanning. zfv, 151-159.

Scaioni, M., 2012. On the estimation of rigid-body transformation for tls registration. In: Proceedings of XXII ISPRS Congress, Technical Commission V, ISPRS Archives-Volumn XXXIX-B5, Melbourne, Australia, 25 Aug - 1 Sep, 2012.

Schürch, P., Densmore, A.L., Rosser, N.J., Lim, M. and McArdell, B.W., 2011. Detection of surface change in complex topography using terrestrial laser scanning: application to the Illgraben debris-flow channel. Earth Surface Processes and Landforms, 36(14), 1847-1859.

Schuhmacher, S. and Böhm, J., 2005. Georeferencing of terrestrial laser scanner data for applications in architectural modelling. In: Proceedings of ISPRS Workshop 3D-ARCH 2005, "Virtual Reconstruction and Visualization of Complex Architectures", ISPRS Archives-Volumn XXXVI-5/W17, Mestre-Venice, Italy, 22-24 August, 2005.

Silvia. E.P. and Olsen M.J., 2012. To level or not to level: laser scanner inclination sensor stability and application. Journal of Surveying Engineering, 138(3), 117-125.

Zhang, J. and Goodchild, M.F., 2002. Uncertainty in Geographical Information. New York: Taylor and Francis. 


\section{Figures:}

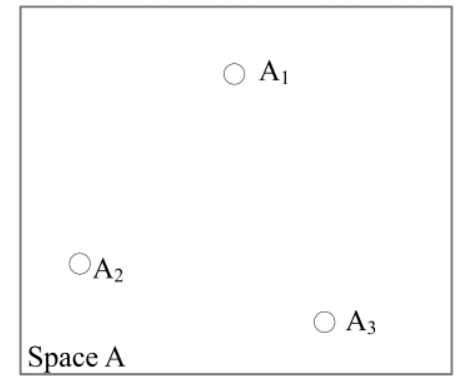

(a)

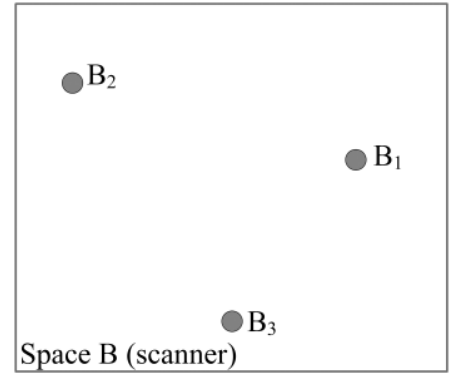

(b)

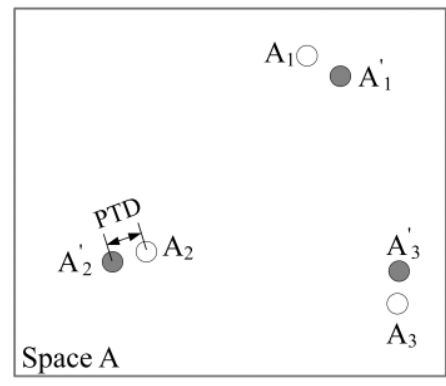

(c)

Figure 1: (a) The targets measured by a scanner for registration or a DGPS/total station for georeferencing; (b) the targets measured by a laser scanner; (c) the targets $\left(B_{1}, B_{2}\right.$ and $\left.B_{3}\right)$ in Space $B$ are transformed into those $\left(\mathrm{A}_{1}^{\prime}, \mathrm{A}_{2}^{\prime}\right.$ and $\left.\mathrm{A}_{3}^{\prime}\right)$ in Space $\mathrm{A}$. 


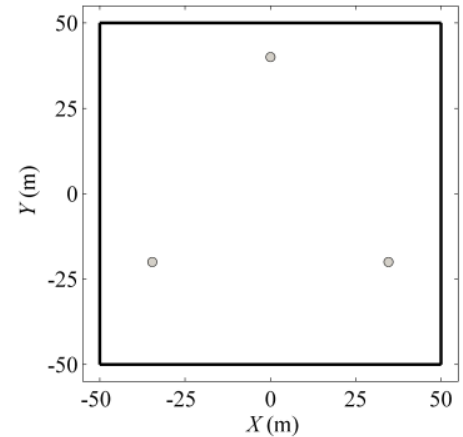

(a)

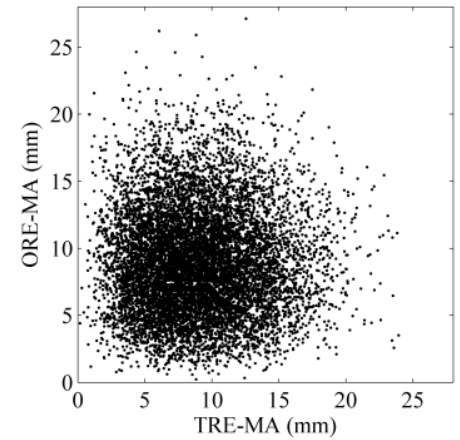

(b)

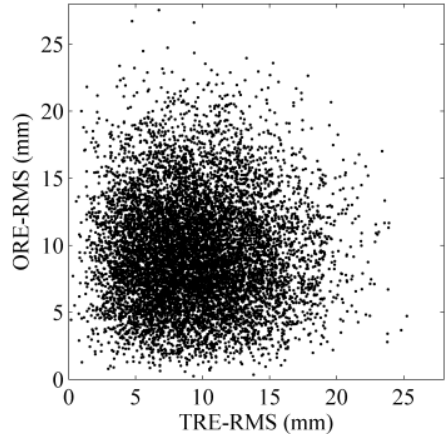

(c)

Figure 2: For the 2D case: (a) The locations of the three targets in the domain of interest; (b) the relation between the TRE-MA and the ORE-MA; (c) the relation between the TRE-RMS and the ORE-RMS. 


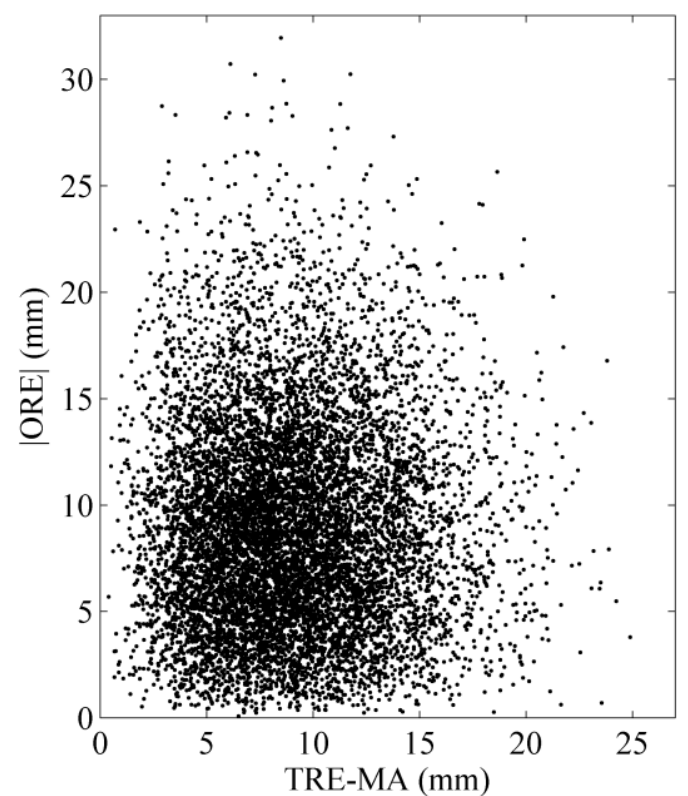

(a)

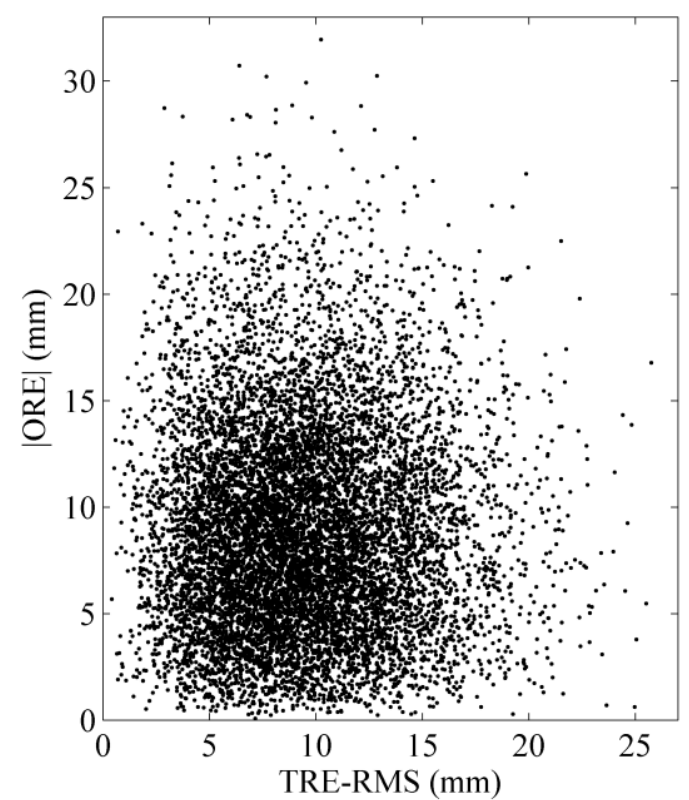

(b)

Figure 3: The relation between the TRE and the |ORE $\mid$ of an individual scan point for the 2D case: (a) in terms of mean absolute error; (b) in terms of root mean squared error. 


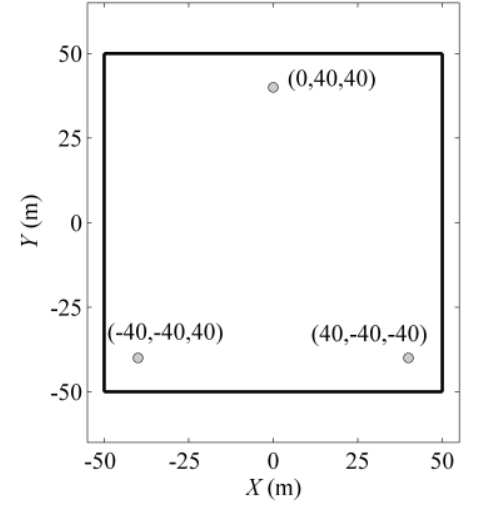

(a)

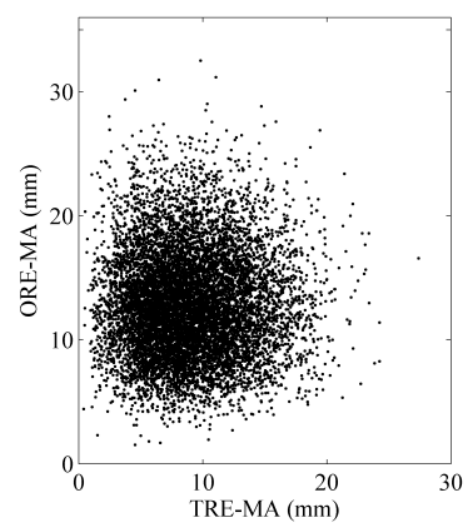

(b)

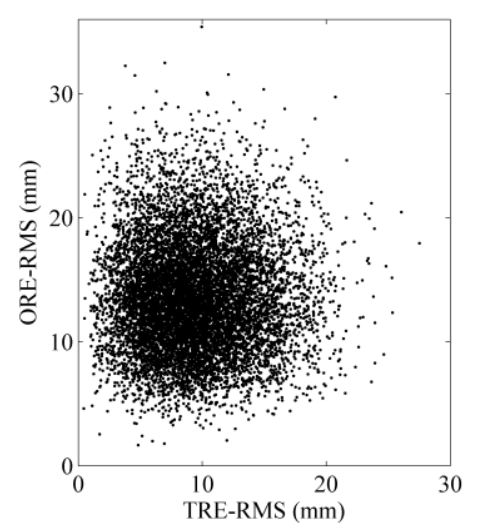

(c)

Figure 4: For the 3D case: (a) The locations of the three targets in the domain of interest; (b) the relation between the TRE-MA and the ORE-MA; (c) the relation between the TRE-RMS and the ORE-RMS. 


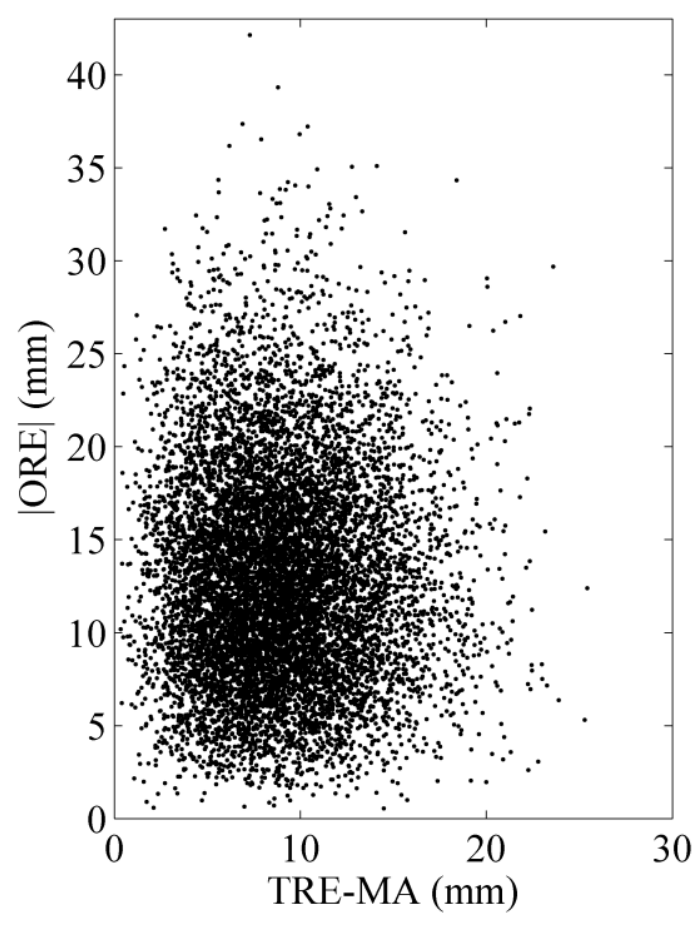

(a)

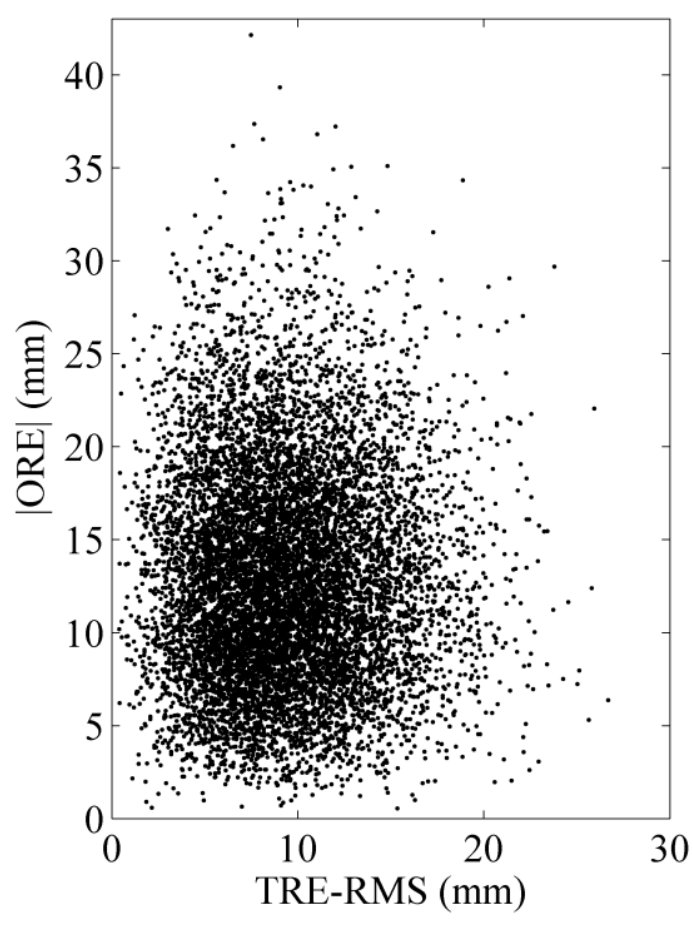

(b)

Figure 5: The relation between the TRE and the |ORE $\mid$ of an individual scan point for the 3D case: (a) in terms of mean absolute error; (b) in terms of root mean squared error. 


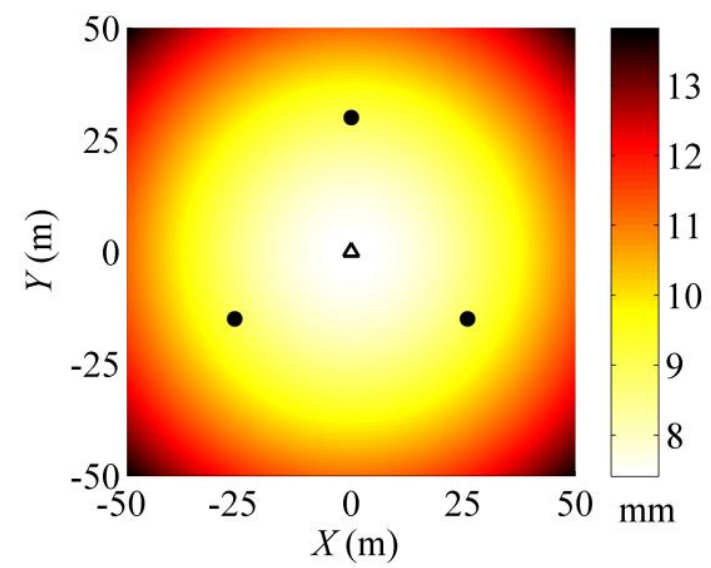

(a)

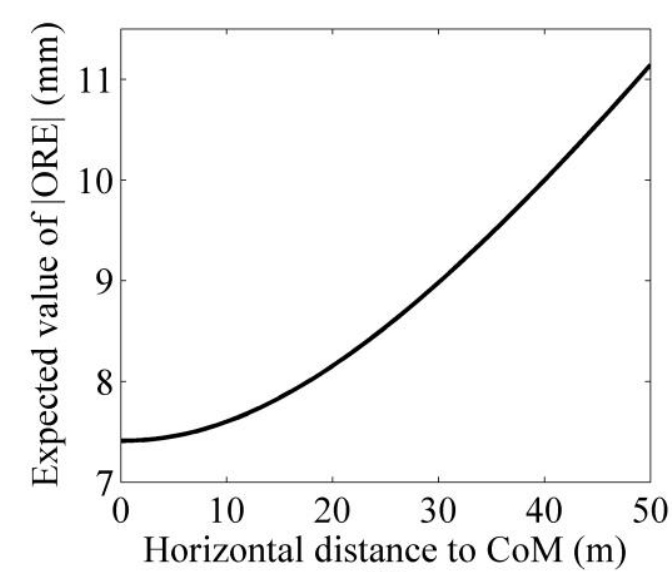

(b)

Figure 6: (a) Spatial distribution of the $\mathrm{E}(|\mathrm{ORE}|)$; (b) the variation of the $\mathrm{E}(|\mathrm{ORE}|)$ with the horizontal distance to the CoM. 


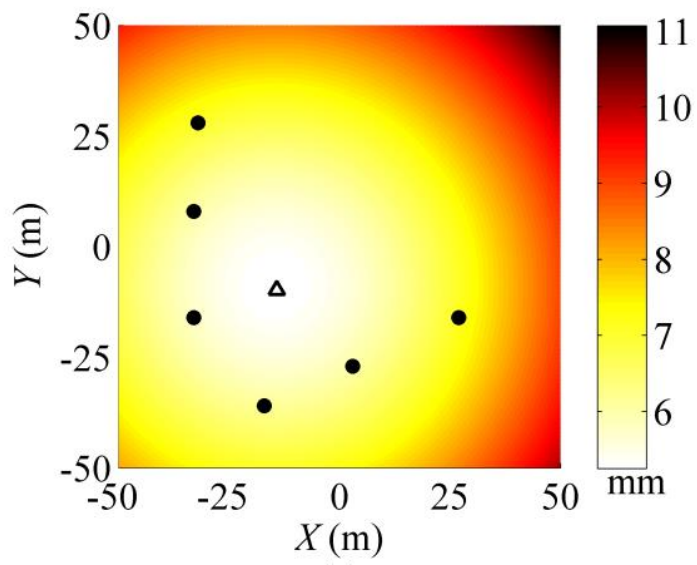

(a)

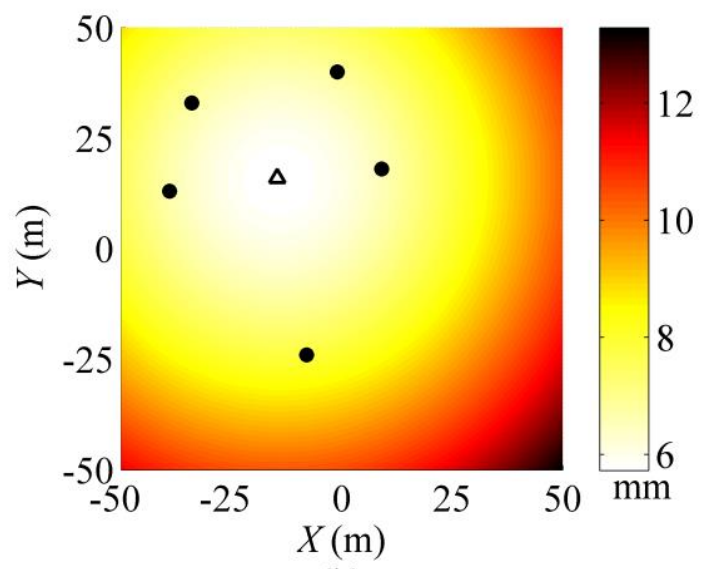

(b)

Figure 7: Spatial pattern and the minimum value of the $\mathrm{E}(|\mathrm{ORE}|)$ for two generic cases (a) and (b); the dot and the triangle represent the targets and their CoM, respectively. 


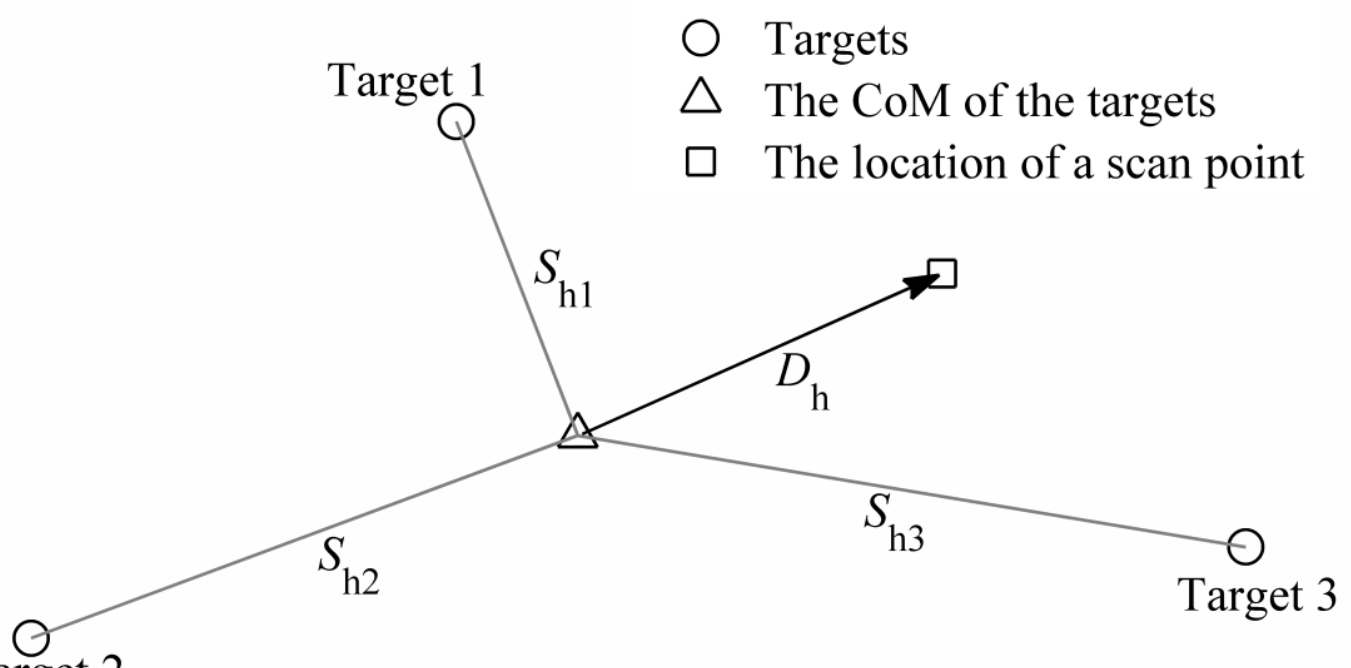

Target 2

Figure 8: The definition of $D$ and $S_{\mathrm{h} i}(i=1,2,3$ in this particular example). 


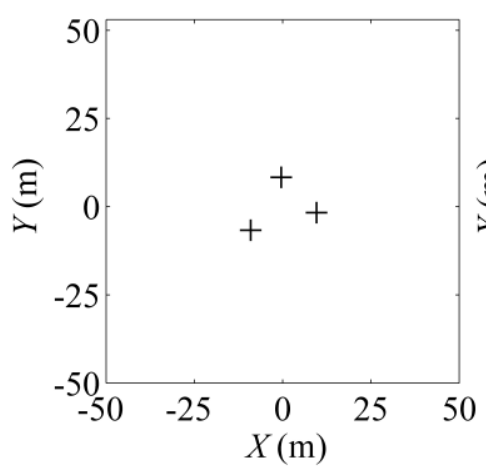

(a)

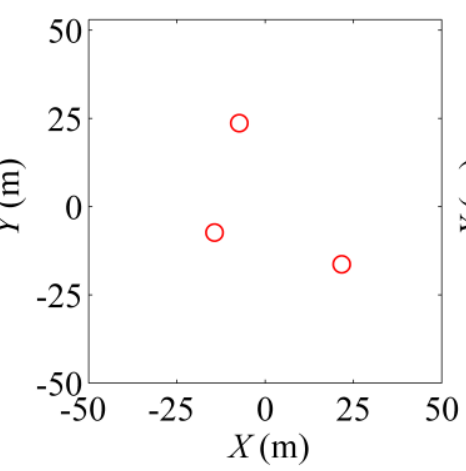

(b)

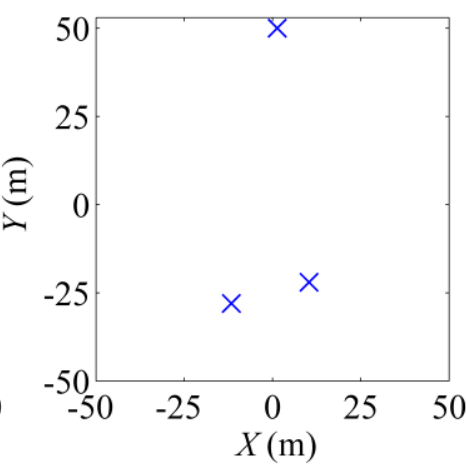

(c)

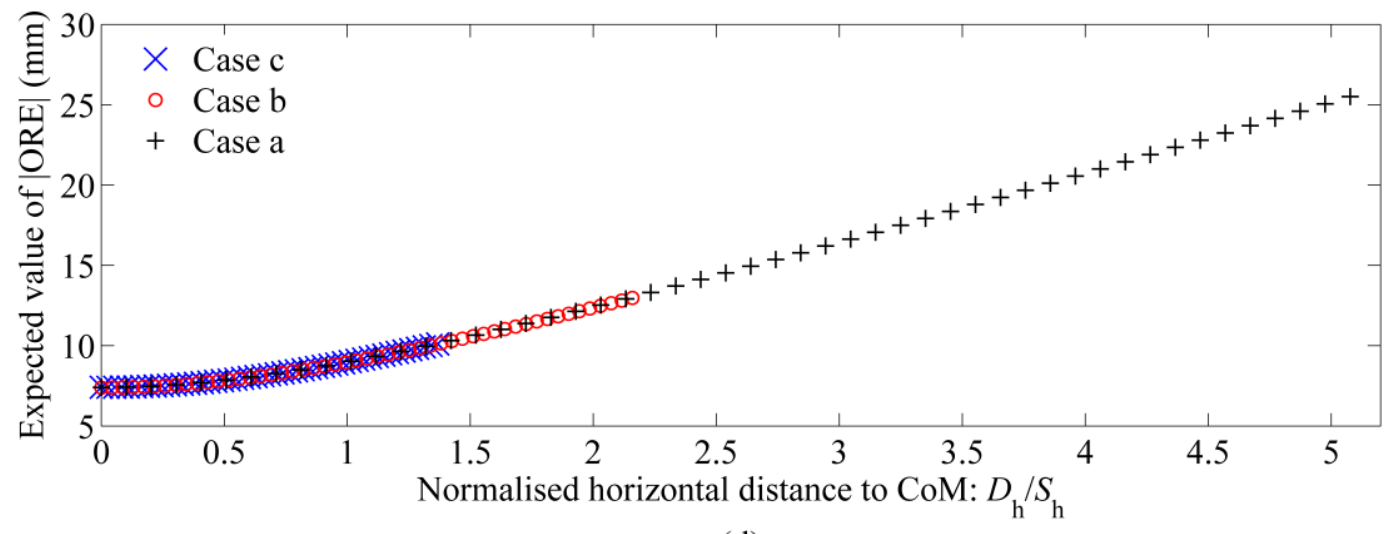

(d)

Figure 9: Normalise horizontal distance to CoM: (a)-(c) three more generic cases; (d) the $\mathrm{E}(|\mathrm{ORE}|$ against the normalised horizontal distance. 


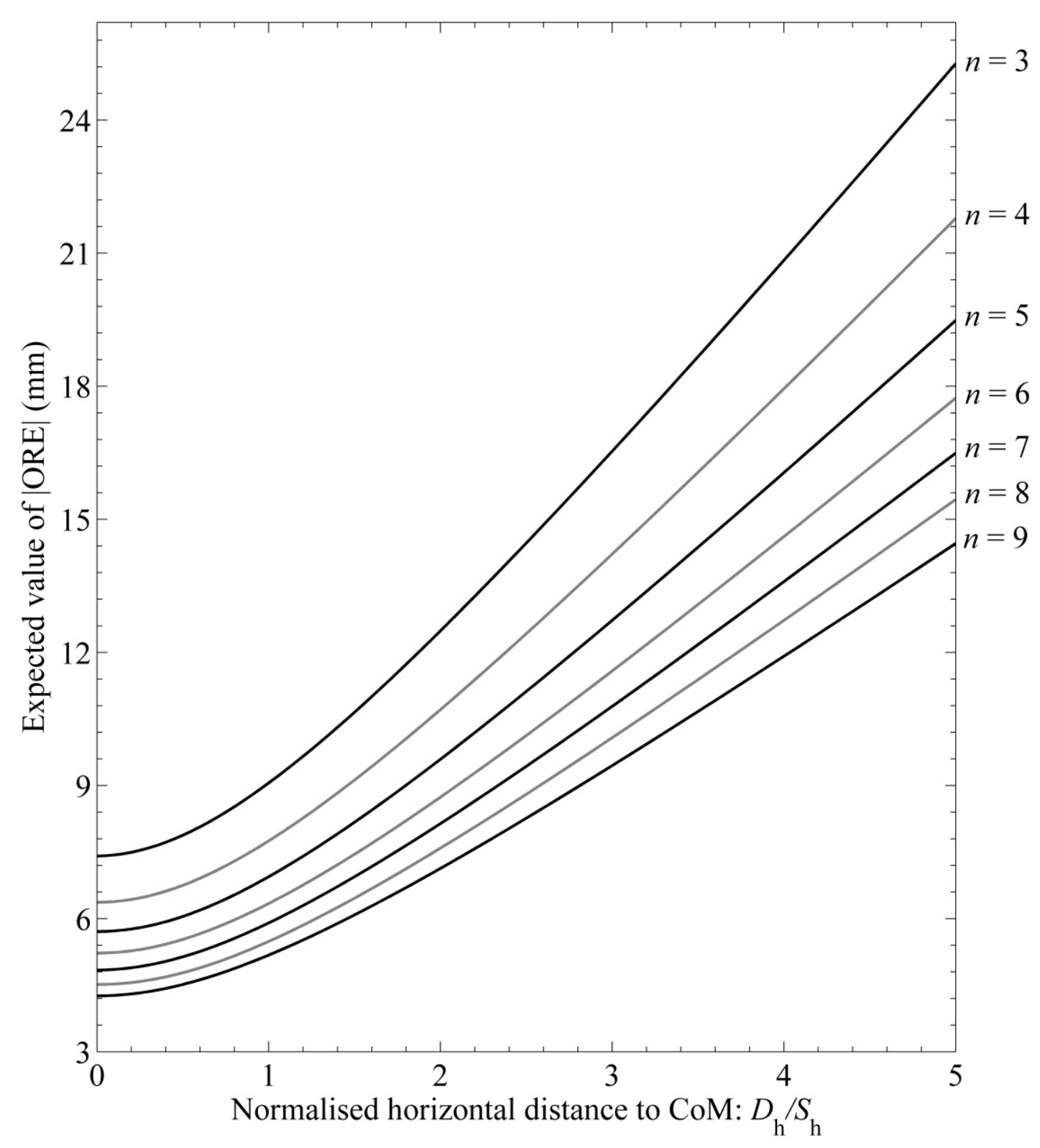

Figure 10: Effect of the number of target constraints $n$ on $\mathrm{E}(|\mathrm{ORE}|)$ 


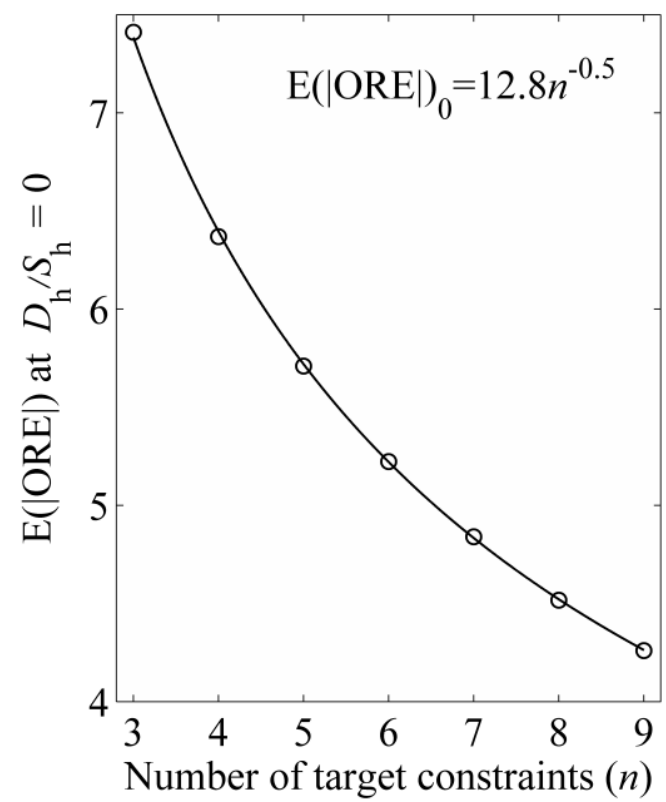

(a)

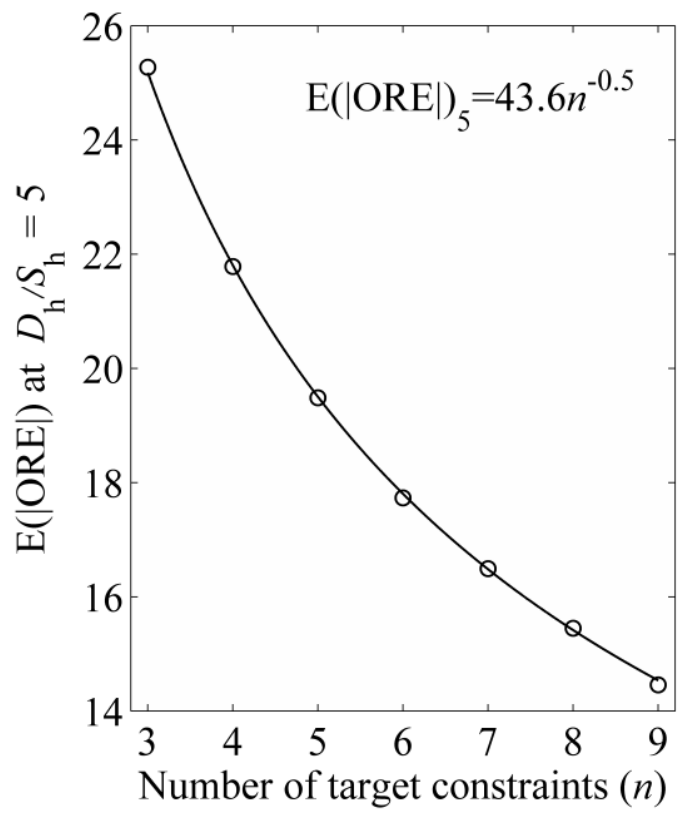

(b)

Figure 11: The effect of the number of target constraints on the E(|ORE $\mid$ ) at: (a) $D_{\mathrm{h}} / S_{\mathrm{h}}=0$ and (b) $D_{\mathrm{h}} / S_{\mathrm{h}}=5$. 


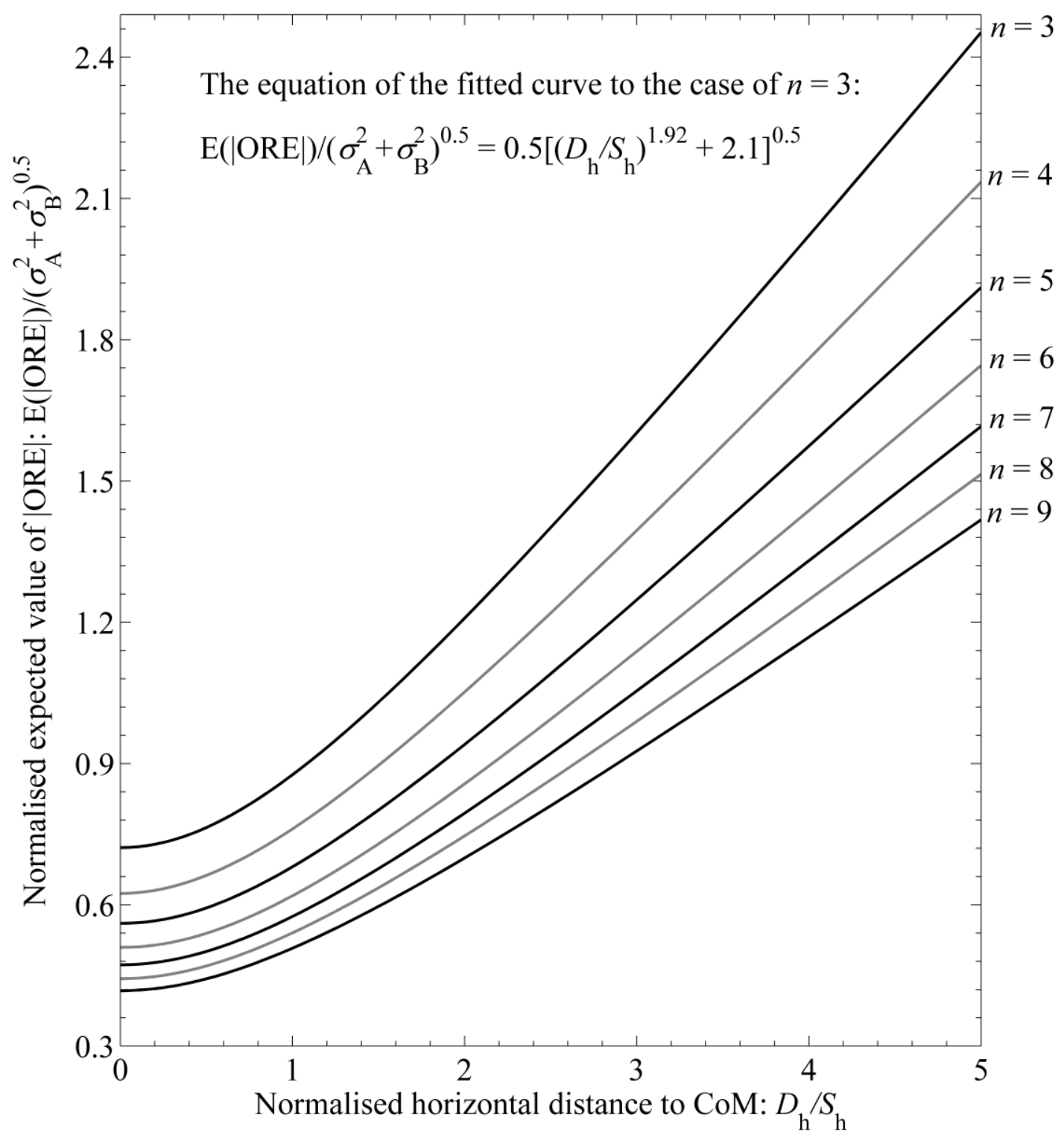

Figure 12: The normalised $\mathrm{E}(|\mathrm{ORE}|)$ against the normalised horizontal distance. 


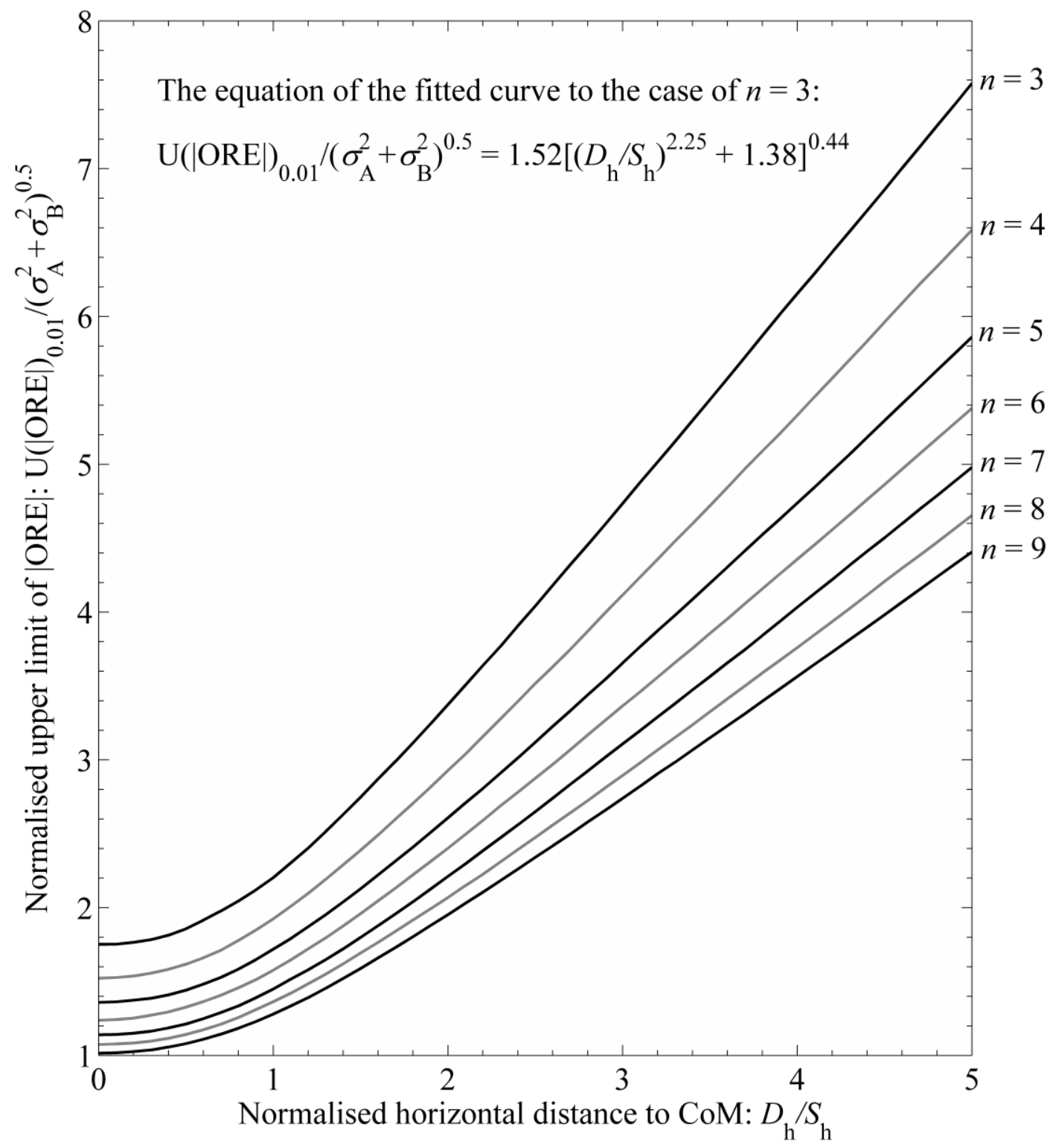

Figure 13: The normalised upper limit of |ORE|. 